\title{
Canonical Coin Systems for Change-Making Problems
}

\author{
Xuan Cai \\ Department of Computer Science and Engineering, Shanghai Jiao Tong University \\ Shanghai 200240, China \\ Email: caixuanfire@sjtu.edu.cn
}

\begin{abstract}
The Change-Making Problem is to represent a given value with the fewest coins under a given coin system. As a variation of the knapsack problem, it is known to be NP-hard. Nevertheless, in most real money systems, the greedy algorithm yields optimal solutions. In this paper, we study what type of coin systems that guarantee the optimality of the greedy algorithm. We provide new proofs for a sufficient and necessary condition for the so-called canonical coin systems with four or five types of coins, and a sufficient condition for non-canonical coin systems, respectively. Moreover, we present an $O\left(\mathrm{~m}^{2}\right)$ algorithm that decides whether a tight coin system is canonical.
\end{abstract}

\section{INTRODUCTION}

The Change-Making Problem comes from the following scenario: in a shopping mall, the cashier needs to make change for many values of money based on some coin system ${ }^{2}$ $\$=\left\langle c_{1}, c_{2}, \cdots, c_{m}\right\rangle$ with $1=c_{1}<c_{2}<\cdots<c_{m}$, where $c_{i}$ denotes the value of the $i$-th type of coin in $\$$. For example, the cent, nickel, dime and quarter are four types of US coins, and the corresponding coin system is $\$=\langle 1,5,10,25\rangle$. Since the reserved coins are limited in reality, the cashier has to handle every exchange with as few coins as possible.

Formally, the Change-Making Problem is to solve the following integer programming problem with respect to a given value $x$.

$$
\begin{array}{cl}
\min & \sum_{i=1}^{m} \alpha_{i} \\
\text { s.t. } & \sum_{i=1}^{m} c_{i} \alpha_{i}=x, \quad c_{i} \geq 0
\end{array}
$$

As usual, we call a feasible solution $\left(\alpha_{1}, \alpha_{2}, \cdots, \alpha_{m}\right)$ of the above integer programming problem a representation of $x$ under $\$$. If this representation satisfies $\sum_{j=1}^{i-1} \alpha_{j} c_{j}<c_{i}$ for $2 \leq i \leq m$, then it is the greedy representation of $x$, denoted by $\operatorname{GRD}_{\$}(x)$, and $\left|\operatorname{GRD}_{\$}(x)\right|=\sum_{i=1}^{m} \alpha_{i}$ is its size. Similarly, we also call the optimal solution $\left(\beta_{1}, \beta_{2}, \cdots, \beta_{m}\right)$ the optimal representation of $x$, denoted by $\mathrm{OPT}_{\$}(x)$, and $\left|\operatorname{OPT}_{\$}(x)\right|=\sum_{i=1}^{m} \beta_{i}$ is its size.

\section{A. Problem Statement}

The Change-Making Problem is NP-hard [8][4][9] by a polynomial reduction from the knapsack problem. There are a large number of pseudo-polynomial exact algorithms [6][10] solving this problem, including the one using dynamic programming [13]. However, the greedy algorithm, as a simpler

\footnotetext{
${ }^{2}$ In this paper, all the variables range over the set $\mathbb{N}$ of natural numbers.
}

approach, can produce the optimal solutions for many practical instances, especially canonical coin systems.

Definition 1: A coin system $\$$ is canonical if $\left|\operatorname{GRD}_{\$}(x)\right|=$ $\left|\mathrm{OPT}_{\$}(x)\right|$ for all $x$.

For example, the coin system $\$=\langle 1,5,10,25\rangle$ is canonical. Accordingly, the cashier can easily create the optimal solution by repeatedly taking the largest coin whose value is no larger than the remaining amount.

Definition 2: A coin system $\$$ is non-canonical if there is an $x$ with $\left|\operatorname{GRD}_{\$}(x)\right|>\left|\operatorname{OPT}_{\$}(x)\right|$, and such $x$ is called a counterexample of $\$$.

Definition 3: A coin system $\$=\left\langle 1, c_{2}, \cdots, c_{m}\right\rangle$ is tight if it has no counterexample smaller than $c_{m}$.

For example, both $\$_{1}=\langle 1,7,10,11\rangle$ and $\$_{2}=\langle 1,7,10,50\rangle$ are non-canonical, and $\$_{1}$ is tight but $\$_{2}$ is not. It is easy to verify that 14 is the counterexample for them, i.e., $\operatorname{GRD}_{\$_{1}}(14)=\operatorname{GRD}_{\$_{2}}(14)=(3,0,0,1)$ and $\mathrm{OPT}_{\$_{1}}(14)=$ $\mathrm{OPT}_{\$_{2}}(14)=(0,2,0,0)$.

Nowadays, canonical coin systems have found numerous applications in many fields, e.g., finance [10], management [3] and computer networks [5]. It is desirable to give a full characterization of them.

\section{B. Related Work}

Chang and Gill [2] were the first to study canonical coin systems. They showed that there must be a counterexample $x$ of the non-canonical $\$=\left\langle 1, c_{2}, \cdots, c_{m}\right\rangle$ such that $c_{3} \leq x<$ $\frac{c_{m}\left(c_{m} c_{m-1}+c_{m}-3 c_{m-1}\right)}{c_{m}-c_{m-1}}$.

Concerning the smallest counterexamples of noncanonical coin systems, Tien and $\mathrm{Hu}$ established the following two important results in [12].

Theorem 1: Let $x$ be the smallest counterexample of the non-canonical coin system $\$=\left\langle 1, c_{2}, \cdots, c_{m}\right\rangle$. Then $\alpha_{i} \beta_{i}=$ 0 for all $i \in[1, m]$ such that $\operatorname{OPT}_{\$}(x)=\left(\alpha_{1}, \alpha_{2}, \cdots, \alpha_{m}\right)$ and $\operatorname{GRD}_{\$}(x)=\left(\beta_{1}, \beta_{2}, \cdots, \beta_{m}\right)$.

Theorem 2: Let $\$_{1}=\left\langle 1, c_{2}, \cdots, c_{m}\right\rangle$ and $\$_{2}=$ $\left\langle 1, c_{2}, \cdots, c_{m}, c_{m+1}\right\rangle$ be two coin systems such that $\$_{1}$ is canonical but $\$_{2}$ is not. Then there is some $k$ such that $k \cdot c_{m}<c_{m+1}<(k+1) \cdot c_{m}$ and $(k+1) \cdot c_{m}$ is a counterexample of $\$ 2$.

These two results not only imply that many coin systems are canonical such as positive integer arithmetic progressions, geometric progressions and the Fibonacci sequence but also are the starting point of a lot of subsequent work. 
Building on Theorem [1, Kozen and Zaks [7] gave a tight range of smallest counterexamples of non-canonical coin systems:

Theorem 3: Let $\$=\left\langle 1, c_{2}, \cdots, c_{m}\right\rangle$ be a coin system. If $\$$ is not canonical, then the smallest counterexample lies in the range

$$
c_{3}+1<x<c_{m-1}+c_{m} .
$$

Furthermore, these bounds are tight.

Moreover, they gave a necessary and sufficient condition of the canonical coin system with three types of coins in [7].

Theorem 4: The coin system $\$=\left\langle 1, c_{2}, c_{3}\right\rangle$ is noncanonical if and only if $0<r<c_{2}-q$ where $c_{3}=q c_{2}+r$ and $r \in\left[0, c_{2}-1\right]$. later.

Here, we provide a proof of this theorem which will be used

\section{Proof of Theorem 4}

$(\Leftarrow)$ Consider the integer $x=c_{2}+c_{3}-1, \operatorname{GRD}_{\$}(x)=\left(c_{2}-\right.$ $1,0,1)$. Since $(r-1, q+1,0)$ is a representation of $x$, we have $\left|\operatorname{OPT}_{\$}(x)\right| \leq r+q$. By the precondition $0<$ $r<c_{2}-q$, it is easy to see $\left|\mathrm{OPT}_{\$}(x)\right|<\left|\operatorname{GRD}_{\$}(x)\right|$. Thus, $c_{3}+c_{2}-1$ is a counterexample of $\$$ and it is non-canonical.

$\Rightarrow$ Since $\$$ is non-canonical, let $x$ be the smallest counterexample. By Theorem $3, x \in\left[c_{3}+2, c_{2}+c_{3}-1\right]$. Without loss of generality, let $\operatorname{GRD}_{\$}(x)=(e, 0,1)$ and $\mathrm{OPT}_{\$}(x)=(0, k, 0)$ with $e \in\left[1, c_{2}-1\right]$. Then we have $x=c_{3}+e=k c_{2}$, i.e., $q=k-1<e$ and $r=c_{2}-e \geq 1$. Thus, $0<r<c_{2}-q$.

Pearson [11] proved the following theorem that characterizes the smallest counterexample of the non-canonical coin system.

Theorem 5: Let $x$ be the smallest counterexample of the non-canonical coin system $\$=\left\langle 1, c_{2}, \cdots, c_{m}\right\rangle$. If $\operatorname{OPT}_{\$}(x)=(\underbrace{0, \cdots, 0}_{0}, \beta_{l}, \cdots, \beta_{r}, \underbrace{0, \cdots, 0}_{0})$ with $\beta_{l}, \beta_{r}>0$, then $\operatorname{GRD}_{\$}\left(c_{r+1}-1\right)=\left(\alpha_{1}, \cdots, \alpha_{l-1}, \beta_{l}-\right.$ $1, \beta_{l+1}, \cdots, \beta_{r}, \underbrace{0, \cdots, 0}_{0})$.

Based on this theorem, he gave an $O\left(\mathrm{~m}^{3}\right)$ algorithm to decide whether a coin system $\$=\left\langle 1, c_{2}, \cdots, c_{m}\right\rangle$ is canonical.

Recently, Niewiarowska and Adamaszek [1] investigate the structure of canonical coin systems and present a series of sufficient conditions of non-canonical coin systems.

\section{Our Contribution}

In this paper, we study canonical coin systems for the Change-Making Problem and obtain the following results.

- We give an easy proof for a sufficient and necessary condition of canonical coin systems with four or five types of coins.

- We provide a new proof for natural sufficient condition of non-canonical coin systems.

- We present an $O\left(\mathrm{~m}^{2}\right)$ algorithm that decides whether a tight coin system is canonical.
The rest of this paper is organized as follows. In Section 2 , we study canonical coin systems with four types of coins. In Section 3, we extend the study to canonical coin systems with five types of coins. Section 4 introduces tight canonical coin systems and Section 5 presents an $O\left(\mathrm{~m}^{2}\right)$ algorithm that decides whether a tight coin system is canonical. Finally, in Section 6, we address some of the questions left open and discuss future work.

\section{Coin System with Four Types of CoInS}

In this section, we study canonical coin systems with four types of coins and give a full characterization of them based on Theorem 4

Theorem 6: A coin system $\$=\left\langle 1, c_{2}, c_{3}, c_{4}\right\rangle$ is noncanonical if and only if $\$$ satisfies exactly one of the following conditions:

1) $\left\langle 1, c_{2}, c_{3}\right\rangle$ is non-canonical.

2) $\left|\operatorname{GRD}_{\$}\left((k+1) \cdot c_{3}\right)\right|>k+1$ with $k \cdot c_{3}<c_{4}<(k+1) \cdot c_{3}$.

The proof is based on an analysis of the coin system $\left\langle 1, c_{2}, c_{3}\right\rangle$. If $\left\langle 1, c_{2}, c_{3}\right\rangle$ is canonical, we can decide whether $\$$ is canonical by Theorem 2 Otherwise, the following lemma covers the remaining case.

Lemma 1: Let $\$=\left\langle 1, c_{2}, c_{3}\right\rangle$ be a coin system with $c_{3}=$ $q c_{2}+r$. If $\$$ is non-canonical, then the coin system $\$^{\prime}=$ $\left\langle 1, c_{2}, c_{3}, c_{4}\right\rangle$ is also non-canonical.

Proof: Since $\$$ is non-canonical, we can find the smallest counterexample $x \in\left[c_{3}+2, c_{2}+c_{3}-1\right]$ by Theorem 3 . Assume that there is some $c_{4}>c_{3}$ such that $\mathbb{S}^{\prime}=\left\langle 1, c_{2}, c_{3}, c_{4}\right\rangle$ is canonical. We will deduce a contradiction based on the analysis of $x$.

- If $x<c_{4}$, then $x$ is a counterexample of $\$^{\prime}$, a contradiction.

- Otherwise, $x \geq c_{4}$. It is easy to see $(0,1,1,0)$ is a representation of $c_{2}+c_{3}$, and $\left|\mathrm{OPT}_{\Phi^{\prime}}\left(c_{2}+c_{3}\right)\right|=2$ for $c_{4} \leq x \leq c_{2}+c_{3}-1$. By the above assumption, we know $\delta=c_{2}+c_{3}-c_{4}$ must be a coin, and here $\delta=1$. Hence, $x=c_{4}=c_{2}+c_{3}-1$. By the proof of Theorem 4, we have $x=k c_{2}=c_{2}+c_{3}-1$, i.e., $r=1$ and $c_{3}=q c_{2}+1$ and $c_{4}=q c_{2}+c_{2}$. Thus,

$$
\$^{\prime}=\left\langle 1, c_{2}, q c_{2}+1, q c_{2}+c_{2}\right\rangle .
$$

For the integer $2 q c_{2}+c_{2}-1$, it is easy to see that $\left(c_{2}-\right.$ $3,0,2,0)$ is a representation under $\$^{\prime}$, and $\mathrm{GRD}_{\mathbb{S}^{\prime}}\left(2 q c_{2}\right.$ $\left.+c_{2}-1\right)=\left(c_{2}-1, q-1,0,1\right)$. Hence, we have $\mid\left(c_{2}-\right.$ $3,0,2,0)|<| \operatorname{GRD}_{\$^{\prime}}\left(2 q c_{2}+c_{2}-1\right) \mid$, that is, $2 q c_{2}+c_{2}-1$ is a counterexample of $\$^{\prime}$, a contradiction.

Moreover, we prove the following Theorem 7 in which the coin system with three types of coins plays a somewhat surprising role.

Theorem 7: If a coin system $\$_{1}=\left\langle 1, c_{2}, c_{3}\right\rangle$ is noncanonical, then the coin system $\$_{2}=\left\langle 1, c_{2}, c_{3}, \cdots, c_{m}\right\rangle$ is also non-canonical for $m \geq 4$.

Actually, we can prove the following stronger result on the counterexamples of non-canonical systems with more than three types of coins. 
Theorem 8: If the coin system $\$_{1}=\left\langle 1, c_{2}, c_{3}\right\rangle$ is noncanonical, then the coin system $\$_{2}=\left\langle 1, c_{2}, c_{3}, \cdots, c_{m}\right\rangle$ is non-canonical and there exists some counterexample $x<$ $c_{m}+c_{3}$ for $m \geq 4$.

The proof is based on an induction on $m$ and an exhaustive case-by-case analysis of $c_{k+1}<c_{k}+c_{3}$. The long proof is placed in the Appendix.

\section{Coin System With FIVE Types OF CoIns}

In this section, we give a full characterization of canonical coin systems with five types of coins.

Theorem 9: A coin system $\$=\left\langle 1, c_{2}, c_{3}, c_{4}, c_{5}\right\rangle$ is noncanonical if and only if $\$$ satisfies exactly one of the following conditions:

1) $\left\langle 1, c_{2}, c_{3}\right\rangle$ is non-canonical.

2) $\$ \neq\left\langle 1,2, c_{3}, c_{3}+1,2 c_{3}\right\rangle$.

3) $\left|\operatorname{GRD}_{\$}\left((k+1) \cdot c_{4}\right)\right|>k+1$ with $k \cdot c_{4}<c_{5}<(k+1) \cdot c_{4}$.

The proof of this theorem is similar to that of Theorem 6 except for the second item $\$ \neq\left\langle 1,2, c_{3}, c_{3}+1,2 c_{3}\right\rangle$. We actually need to prove the following theorem.

Theorem 10: The coin system $\$_{1}=\left\langle 1, c_{2}, c_{3}, c_{4}\right\rangle$ is noncanonical and the coin system $\$_{2}=\left\langle 1, c_{2}, c_{3}, c_{4}, c_{5}\right\rangle$ is canonical if and only if $c_{3}>3$ and $\$_{2}=\left\langle 1,2, c_{3}, c_{3}+1,2 c_{3}\right\rangle$.

The proof is based on an exhaustive case-by-case analysis of the smallest counterexamples of some coin systems. The proof can be found in the Appendix.

\section{Tight Coin System}

For a coin system $\$=\left\langle 1, c_{2}, \cdots, c_{m}, c_{m+1}\right\rangle$, once there is an untight subsystem $\left\langle 1, c_{2}, \cdots, c_{i}\right\rangle$ with $i \leq m+1$, $\$$ is clearly non-canonical. Therefore, it is necessary to decide whether a tight coin system is canonical.

Theorem 11: Let $\$_{1}=\left\langle 1, c_{2}, c_{3}\right\rangle, \$_{2}=\left\langle 1, c_{2}, c_{3}, \cdots, c_{m}\right\rangle$ and $\$_{3}=\left\langle 1, c_{2}, c_{3}, \cdots, c_{m}, c_{m+1}\right\rangle$ be three tight coin systems such that $\$_{1}$ is canonical but $\$_{2}$ is not. If $\$_{3}$ is non-canonical, then there is a counterexample $x=c_{i}+c_{j}>c_{m+1}$ of $\$_{3}$ with $1<c_{i} \leq c_{j} \leq c_{m}$.

To establish Theorem 11, we first prove Lemma 2. Here, we define $c_{0}=0$ and $d_{i}:=c_{i}-c_{i-1}$ with $1 \leq i \leq m+1$.

Lemma 2: Let $\$_{1}=\left\langle 1, c_{2}, c_{3}\right\rangle, \$_{2}=\left\langle 1, c_{2}, c_{3}, \cdots, c_{m}\right\rangle$ and $\$_{3}=\left\langle 1, c_{2}, c_{3}, \cdots, c_{m}, c_{m+1}\right\rangle$ be three tight coin systems. $\$_{1}$ is canonical but both $\$_{2}$ and $\$_{3}$ are not. If any $c_{m}+c_{i}>$ $c_{m+1}$ is not the counterexample of $\$_{3}$ with $1<c_{i} \leq c_{m}$, then $d_{m+1}=\max \left\{d_{i} \mid 1 \leq i \leq m+1\right\}$.

Proof: Assume that there is some $d_{j+1}>d_{m+1}$ with $0<j<m$. Without loss of generality, let $d_{j+1}=d_{m+1}+\kappa$ with $0<\kappa<d_{j+1}$.

For $c_{j+1}+c_{m}$, we have $c_{j+1}+c_{m}=c_{j}+d_{j+1}+c_{m}=$ $c_{j}+\kappa+c_{m+1}$. Since $c_{j}+\kappa \in\left(c_{j}, c_{j+1}\right)$, we have that $c_{j+1}+c_{m}$ is a counterexample of $\$_{3}$. This is a contradiction.

Proof of Theorem 11. Assume that any $x=c_{i}+c_{j}>$ $c_{m+1}$ with $1<c_{i} \leq c_{j} \leq c_{m}$ is not the counterexample of $\$_{3}$. By Lemma 2 and the assumption, $d_{m+1}=\max \left\{d_{i} \mid 1 \leq\right.$ $i \leq m+1\}$. For simplicity, we introduce some notations.

- $x=c_{m+1}+\delta$ is the smallest counterexample of $\$_{3}$.
- $c_{s}$ is the largest coin used in all the optimal representations of $x$ with $s \leq m$.

- $c_{l}$ and $c_{h}$ are the smallest coin and the largest coin used in the optimal representation of $x-c_{s}$ respectively.

Thus, we have $c_{l}>x-c_{s+1}$ by definition of $c_{s}$.

(1) If $s \leq m-1$, then $c_{l}>x-c_{m}=\delta+d_{m+1}$.

- If $c_{s}+c_{l}<x$, then $\mathrm{OPT}_{\$_{3}}(x)$ uses one coin $c_{h}$ besides a coin $c_{l}$ and a coin $c_{s}$. Since $\mid \operatorname{GRD}_{\$_{3}}(x-$ $\left.c_{h}\right)|=| \mathrm{OPT}_{\$_{3}}\left(x-c_{h}\right) \mid$ and $x-c_{h} \geq c_{s}+c_{l}>c_{s+1}$, we have $c_{s+1}$ appears in $\mathrm{OPT}_{\$_{3}}(x)$, a contradiction.

- Otherwise, $c_{s}+c_{l}=x>c_{m+1}$. By the assumption, there is $c_{m+1}+c_{i}=c_{s}+c_{l}$, a contradiction.

(2) Otherwise, $s=m$.

- If $c_{h}+c_{m}>c_{m+1}$, there exists $c_{m+1}+c_{i}=c_{h}+c_{s}$ by the assumption. Thus, we can get a new representation of $x$ replacing $c_{h}$ and $c_{m}$ with $c_{m+1}$ and $c_{i}$ in $\mathrm{OPT}_{\$_{3}}(x)$. It is easy to see this new representation uses the coin $c_{m+1}$ and remains optimality, a contradiction.

- If $c_{h}+c_{m}<c_{m+1}$, then $\mathrm{OPT}_{\$_{3}}(x)$ uses one coin $c_{l}$ besides a coin $c_{h}$ and a coin $c_{m}$. Since $s=m$, we have $c_{l}>x-c_{m+1}$, i.e., $\delta<c_{l} \leq c_{h}<d_{m+1}$. Thus, $c_{m}+\delta \in\left(c_{m}, c_{m+1}\right)$. It is easy to see $\left|\operatorname{GRD}_{\$_{3}}(x)\right|>$ $\left|\mathrm{OPT}_{\$_{3}}(x)\right|=\left|\mathrm{GRD}_{\$_{2}}(x)\right|$. By the assumption, there is $c_{m+1}+c_{i}=2 c_{m}$. Then we have

$$
\begin{aligned}
\left|\operatorname{GRD}_{\$_{3}}\left(c_{m}+\delta\right)\right| & =\left|\operatorname{GRD}_{\$_{3}}(x)\right| \\
& >1+\left|\operatorname{GRD}_{\$_{3}}\left(c_{m}+\delta-c_{i}\right)\right|
\end{aligned}
$$

It implies $c_{m}+\delta$ is a counterexample of $\$_{3}$, a contradiction.

- If $c_{h}+c_{m}=c_{m+1}$, then we can get a new representation of $x$ replacing $c_{h}$ and $c_{m}$ with $c_{m+1}$ in $\mathrm{OPT}_{\$_{3}}(x)$. It is easy to see this new representation has the smaller size, a contradiction.

\section{THE Algorithm}

In this section, we present an $O\left(\mathrm{~m}^{2}\right)$ algorithm that decides whether a tight coin system $\$=\left\langle 1, c_{2}, \cdots, c_{m}, c_{m+1}\right\rangle$ with $m \geq 5$ is canonical. By Theorem [11, we have if $\$$ is noncanonical, then there is a counterexample that is the sum of two coins. 


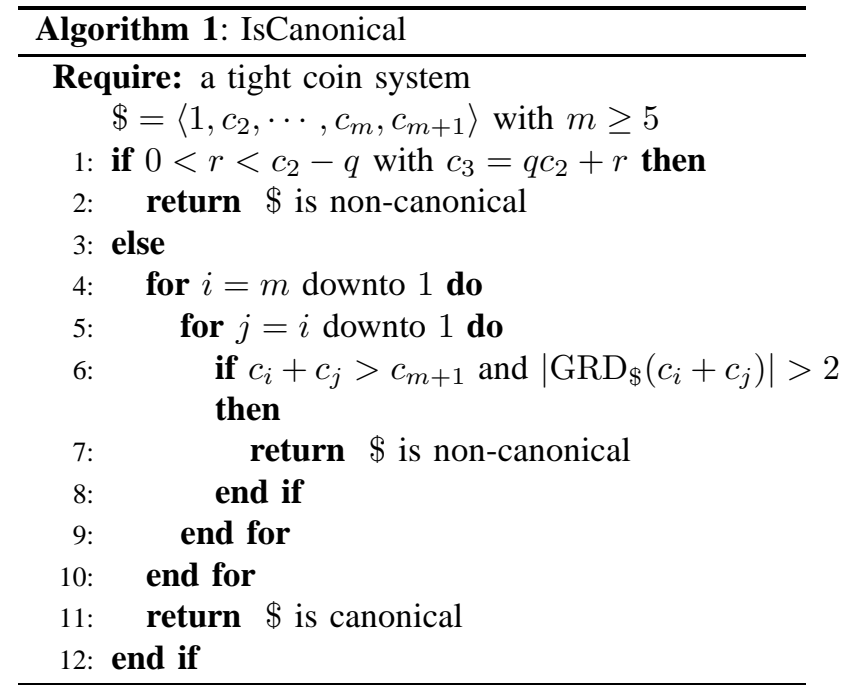

In addition, we observe this algorithm can deal with most tight coin systems in $2 m$ steps, except a small number of non-canonical coin systems, and they are almost arithmetic progressions, for example,

$$
\$=\langle 1,2, \cdots, 12,14,15, \cdots, 20,21,24,25,26,28,29,30,39\rangle
$$

Moreover, we can characterize the smallest counterexample of such non-canonical coin system.

Lemma 3: Let $\$_{1}=\left\langle 1, c_{2}, c_{3}\right\rangle, \$_{2}=\left\langle 1, c_{2}, c_{3}, \cdots, c_{m}\right\rangle$ and $\$_{3}=\left\langle 1, c_{2}, c_{3}, \cdots, c_{m}, c_{m+1}\right\rangle$ be three tight coin systems such that $\$_{1}$ is canonical but $\$_{2}$ and $\$_{3}$ are not. If $\$_{3}$ has no counterexample $x$ such that $x=c_{m}+c_{i}>c_{m+1}$ or $x=$ $c_{m-1}+c_{j}>c_{m+1}$ with $1<c_{i}, c_{j} \leq c_{m}$, then the smallest counterexample of $\$_{3}$ is the sum of two coins.

Proof: Modifying the proof of Theorem 11 slightly, it is easy to get this proof.

\section{CONCLUSION}

In this paper, we have given an $O\left(\mathrm{~m}^{2}\right)$ algorithm that decides whether a tight coin system is canonical. Although our algorithm can only handle tight coin systems, it is more efficient than Pearson's algorithm. As some future work, we expect to obtain an $O(m)$ algorithm for tight canonical coin systems and an $o\left(\mathrm{~m}^{3}\right)$ general algorithm.

We have shown a sufficient and necessary condition of canonical coin systems with four or five types of coins by a novel method. Meantime, we have also obtained a sufficient condition of non-canonical coin systems. Many algorithm including Pearson's can benefit from it. However, it is still left open to give full characterizations of canonical coin systems with more than five types of coins. It is a challenge to explore the corresponding necessary condition in the future.

\section{ACKNOWLEDGMENT}

We would like to thank Yiyuan Zheng for his valuable suggestions and discussion.

\section{REFERENCES}

[1] M. Adamaszek and A. Niewiarowska. "Combinatorics of the Change-Making Problem,' http://arxiv.org/PS_cache/arxiv/pdf/0801/0801.0120v2.pdf

[2] S. K. Chang and A. Gill. "Algorithmic Solution of the ChangeMaking Problem," Journal of the Association for Computing Machinery, 17(1):113-122, 1970.

[3] S. Eilon. "Appication of the Knapsack Model for Budgeting," Omega, 15(6):489-494, 1987

[4] M. R. Garey and D. S. Johnson. Computers and Intractability: a Guide to the Theory of NP-completeness. W. H. Freeman, 1979.

[5] M. Gerla and L. Kleinrock. "On the Topological Design of Distributed Computer Networks," IEEE Transactions on Communications, 25:48-60, 1977.

[6] P. J. Kolesar. "A Branch and Bound Algorithm for the Knapsack Problem," Management Science, 13(9):723-735, 1967.

[7] D. Kozen and S. Zaks. "Optimal Bounds for the Change-Making Problem," Theoretical Computer Science, 123(2):377-388, 1994.

[8] G. S. Lueker. "Two NP-complete Problems in Nonnegative Integer Programming," Report 178, Computer Science Laboratory, Princeton University, 1975.

[9] M. J. Magazine, G. L. Nemhauser and L. E. Trotter. "When the Greedy Solution Solves a Class of Knapsack Problem," Operations Reseach, 23(2):207-217, 1975

[10] G. L. Nemhauser and Z. Ullman. "Discrete Dynamic Programming and Capital Allocation," Management Science, 15(9):494-505, 1969.

[11] D. Pearson. "A Polynomial-time Algorithm for the Change-Making Problem," Operations Reseach Letters, 33(3):231-234, 2005.

[12] B. N. Tien and T. C. Hu. "Error Bounds and the Applicability of the Greedy Solution to the Coin-Changing Problem," Operations Reseach, 23(3):404-418, 1977.

[13] J. W. Wright. "The Change-Making Problem," Journal of the Association for Computing Machinery, 22(1):125-128, 1975.

\section{APPENDIX}

Proof of Theorem 8 . The proof is based on the induction on $m$ and the exhaustive case-by-case analysis of $c_{m+1}<$ $c_{m}+c_{3}$.

The result is trivial for $m=4$ by Lemma 1 and Theorem 3 Now assume that $\$_{2}=\left\langle 1, c_{2}, c_{3}, \cdots, c_{k}\right\rangle$ is non-canonical and there exists some counterexample $x<c_{k}+c_{3}$.

Then we will check $\$_{2}^{\prime}=\left\langle 1, c_{2}, c_{3}, \cdots, c_{k}, c_{k+1}\right\rangle$ based on a detailed analysis of the various cases for $c_{k+1}$. Here, we only consider the non-trivial cases as follows:

- $x>c_{k}$. Otherwise, $x$ is also a counterexample of $\$_{2}^{\prime}$ and $x<c_{k+1}+c_{3}$.

- $c_{i}-c_{i-1}<c_{3}$ for $i \in[4, k+1]$. Otherwise, there must be some integer $y$ such that $y$ is a counterexample of $\$_{2}^{\prime}$ and $y<c_{k+1}+c_{3}$ by the previous assumption.

- Once $c_{i}+c_{j} \in\left(c_{s}, c_{s+1}\right)$ and $c_{i}+c_{j}<c_{k+1}+c_{3}$ with $i \leq$ $j<s \leq k+1$, we have $c_{i}+c_{j}-c_{s} \in\left\{1, c_{2}, c_{3}, \cdots, c_{m}\right\}$. Otherwise, $c_{i}+c_{j}$ is a counterexample of $\$_{2}^{\prime}$ and $c_{i}+c_{j}<$ $c_{k+1}+c_{3}$.

Next, we will analyze $c_{k+1}<c_{k}+c_{3}$ exhaustively, and either find a counterexample of $\$_{2}^{\prime}$ or exclude a case for a contradiction.

(1) If $c_{k+1}=c_{k}+1$, then $c_{k}+c_{2}$ is a counterexample of $\$_{2}^{\prime}$ and $c_{k}+c_{2}<c_{k+1}+c_{3}$.

(2) If $c_{k+1}=c_{k}+\kappa$ with $\kappa \in\left(1, c_{2}\right)$, then $c_{k}+c_{2} \in$ $\left(c_{k+1}, c_{k+1}+c_{2}-1\right)$ and $c_{k}+c_{3} \in\left(c_{k+1}, c_{k+1}+c_{2}-1\right)$. Thus, we have

$$
\begin{aligned}
& c_{k}+c_{2}-c_{k+1}=c_{2}-\kappa \in\{1\} \\
& c_{k}+c_{3}-c_{k+1}=c_{3}-\kappa \in\left\{1, c_{2}\right\}
\end{aligned}
$$


It is easy to see $2 c_{2}=c_{3}+1$ for $c_{3}-\kappa>c_{2}-\kappa$. Thus, $\$_{1}$ is canonical by Theorem 4 a contradiction.

(3) If $c_{k+1}=c_{k}+c_{3}-\kappa$ with $\kappa \in\left(0, c_{3}-c_{2}\right]$, then $c_{k}+c_{3} \in$ $\left(c_{k+1}, c_{k+1}+c_{3}-c_{2}\right]$ and $c_{k}+c_{4} \in\left(c_{k+1}, c_{k+1}+c_{4}-c_{2}\right]$. Thus, we have

$$
\begin{aligned}
& c_{k}+c_{3}-c_{k+1}=\kappa \in\left\{1, c_{2}\right\} \\
& c_{k}+c_{4}-c_{k+1}=c_{4}-c_{3}+\kappa \in\left\{1, c_{2}, c_{3}\right\}
\end{aligned}
$$

If $\kappa=c_{2}$, then it is easy to see $2 c_{2} \leq c_{3}$ and $c_{4}=2 c_{3}-$ $c_{2} \geq c_{2}+c_{3}$. By Theorem 3 , the smallest counterexample $y$ of $\$_{1}$ is also a counterexample of $\$_{2}^{\prime}$ and $y<c_{k+1}+c_{3}$.

If $\kappa=1$, then $c_{k+1}=c_{k}+c_{3}-1$ and $c_{4}-c_{3}+1 \in\left\{c_{2}, c_{3}\right\}$.

a) If $c_{4}-c_{3}+1=c_{3}$, then $c_{4}=2 c_{3}-1$. Since $\$_{1}$ is non-canonical, by the proof of Theorem 4, we have the smallest counterexample $y=(q+1) c_{2}$ of $\$_{1}$ with $0<r<c_{2}-q$. Replacing $c_{3}$ with $q c_{2}+r$, it is easy to see $c_{4}=2 q c_{2}+2 r-1>y$. Thus, $y$ is a counterexample of $\$_{2}^{\prime}$ and $y<c_{k+1}+c_{3}$.

b) If $c_{4}-c_{3}+1=c_{2}$, then $c_{4}=(q+1) c_{2}+r-1$ with $c_{3}=$ $q c_{2}+r$. Similarly, we have the smallest counterexample $y=(q+1) c_{2}$ of $\$_{1}$ with $0<r<c_{2}-q$.

If $r>1$, then $y$ is a counterexample of $\$_{2}^{\prime}$ and $y<$ $c_{k+1}+c_{3}$.

If $r=1$, then $c_{3}=q c_{2}+1$ and $c_{4}=(q+1) c_{2}$. Since $c_{k-1}>c_{k}-c_{3}$ and $c_{k+1}-c_{k}=c_{3}-1$, we have $c_{k-1}+c_{4} \in\left(c_{k}+c_{2}-1, c_{k}+c_{4}\right)$ and $c_{k}+c_{4}>c_{k+1}$.

- If $c_{k-1}+c_{4} \in\left(c_{k}+c_{2}-1, c_{k+1}\right)$, then $c_{k-1}+c_{4}-c_{k} \in$ $\left\{c_{2}\right\}$, i.e., $c_{k}-c_{k-1}=c_{k+1}-c_{k}=c_{4}-c_{2}=$ $q c_{2}$. Since $c_{k-1}<c_{k-2}+c_{3}$, we have $c_{k-2}+c_{3} \in$ $\left(c_{k-1}, c_{k}\right]$.

If $c_{k-2}+c_{3}=c_{k}$, then $c_{k-1}-c_{k-2}=1$. It is easy to see $c_{k-2}+c_{2} \in\left(c_{k-1}, c_{k}\right)$ is a counterexample of $\$ 2$.

If $c_{k-2}+c_{3}<c_{k}$, then $c_{k-2}+c_{3}-c_{k-1} \in\left\{1, c_{2}\right\}$.

- If $c_{k-2}+c_{3}-c_{k-1}=c_{2}$, then $c_{k-1}-c_{k-2}=$ $c_{3}-c_{2}$.

If $c_{3}-c_{2}>c_{2}$, then $2 c_{3}=c_{4}+c_{2}$. Since $c_{4}=$ $(q+1) c_{2}$, we have $c_{2}=\frac{2}{2-q}$ and $q \geq 1$, i.e., $c_{2}=2$. However, $\$_{1}=\langle 1,2,3\rangle$ is canonical, a contradiction.

If $c_{3}-c_{2}<c_{2}$, then $q=1$ and $c_{3}=c_{2}+1$ and $c_{4}=2 c_{2}$. Thus, $c_{k-2}+c_{2} \in\left(c_{k-1}, c_{k}\right)$ is a counterexample of $\$_{2}^{\prime}$.

- If $c_{k-2}+c_{3}-c_{k-1}=1$, then $c_{k-1}-c_{k-2}=c_{3}-1$.

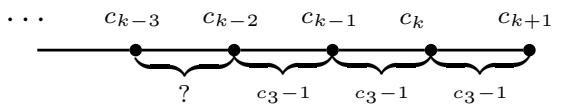

If $c_{k-2}-c_{k-3}<c_{3}-1$, then there is a counterexample $z$ of $\left\langle 1, c_{2}, \cdots, c_{k-3}\right\rangle$ such that $z<$ $c_{k-3}+c_{3} \leq c_{k-1}$ by the previous assumption.
* If $z<c_{k-2}$, then $z<c_{k+1}+c_{3}$ is a counterexample of $\$_{2}^{\prime}$.

* If $c_{k-2} \leq z<c_{k-3}+c_{3}-1$, then $c_{k-2}+z-$ $c_{k-3} \in\left(c_{k-2}, c_{k-1}\right)$, which is a counterexample of $\$_{2}^{\prime}$.

$*$ If $z=c_{k-3}+c_{3}-1$, then $c_{k+1}+c_{3}-1$ is a counterexample of $\$_{2}^{\prime}$.

Otherwise, $c_{k-2}-c_{k-3}=c_{3}-1$. Similarly, we either find a counterexample of $\$_{2}^{\prime}$ or obtain

$$
c_{k-3}-c_{k-4}=\cdots=c_{4}-c_{3}=c_{3}-1=q c_{2} .
$$

Thus, $c_{2}=\frac{1}{1-q}$, a contradiction.

- If $c_{k-1}+c_{4}=c_{k+1}$, then $c_{k}-c_{k-1}=c_{2}$. Since $c_{k-1}+c_{3}=c_{k}+c_{3}-c_{2} \in\left(c_{k}, c_{k+1}\right)$, we have $c_{3}-c_{2} \in\left\{1, c_{2}\right\}$.

- If $c_{3}-c_{2}=c_{2}$, then $c_{3}=2 c_{2}$, a contradiction.

- If $c_{3}-c_{2}=1$, then $c_{3}=c_{2}+1$ and $c_{4}=2 c_{2}$. Since $c_{k-2}+c_{3} \in\left(c_{k-1}, c_{k}\right)$, we have $c_{k-2}+c_{3}-c_{k-1} \in$ $\{1\}$, i.e., $c_{k-1}-c_{k-2}=c_{2}$. Similar to the above proof, we either find a counterexample of $\$_{2}^{\prime}$ or obtain

$$
c_{k-2}-c_{k-3}=\cdots=c_{4}-c_{3}=c_{2} .
$$

It is a contradiction.

- If $c_{k-1}+c_{4} \in\left(c_{k+1}, c_{k+1}+c_{2}\right)$, then $c_{k-1}+c_{4}-$ $c_{k+1} \in\{1\}$. Since $c_{k+1}=c_{k}+c_{3}-1$, we have $c_{k}-c_{k-1}=c_{2}-1$, and $c_{k-1}+c_{3}=c_{k}+c_{3}+1-c_{2}$. Since $c_{3}+1-c_{2} \in\left\{c_{2}\right\}$, we have $c_{3}=2 c_{2}-1$, a contradiction.

Proof of Theorem 10. To show the above theorem, we first need to prove the following lemma.

Lemma 4: Let $\$_{1}=\left\langle 1, c_{2}, c_{3}, c_{2}+c_{3}-1\right\rangle$ and $\$_{2}=$ $\left\langle 1, c_{2}, c_{3}, c_{2}+c_{3}-1, c_{2}+2 c_{3}-2\right\rangle$ be two coin systems such that $\$_{2}$ is canonical but $\$_{1}$ is not. Then the coin system $\$_{3}=\left\langle 1, c_{2}, c_{3}\right\rangle$ is canonical.

Proof: Assume that $\$_{3}$ is non-canonical. By Theorem 3 , there exists the smallest counterexample $y \in\left[c_{3}+2, c_{2}+c_{3}-1\right]$ of $\$_{3}$. Next, we will deduce a contradiction by analyzing $y$ in detail.

(1) If $y \in\left[c_{3}+2, c_{2}+c_{3}-2\right]$, then $y$ is also a counterexample of $\$_{2}$, a contradiction.

(2) Otherwise, $y=c_{2}+c_{3}-1=k c_{2}$ with $1<k<c_{2}$ by the proof of Theorem 4 Thus, we have

$$
\$_{2}=\left\langle 1, c_{2}, k c_{2}-c_{2}+1, k c_{2}, 2 k c_{2}-c_{2}\right\rangle
$$

Since $\$_{1}$ is non-canonical, we have the smallest counterexample $x \in\left[c_{3}+2, c_{2}+2 c_{3}-2\right]$ by Theorem 3

- If $x \in\left[c_{3}+2, c_{2}+2 c_{3}-3\right]$, then $x$ is also a counterexample of $\$_{2}$, a contradiction. 
- Otherwise, $x=c_{2}+2 c_{3}-2=2 k c_{2}-c_{2}$. By Theorem 1 , we have

$$
\begin{aligned}
& \operatorname{GRD}_{\$_{1}}(x)=(0, k-1,0,1) \\
& \operatorname{OPT}_{\Phi_{1}}(x)=\left(\beta_{1}, 0, \beta_{3}, 0\right) \quad \text { with } \beta_{3} \leq 2
\end{aligned}
$$

- If $\beta_{3} \leq 1$, then $\beta_{1}>k-1$. It is easy to see $\left|\mathrm{OPT}_{\$_{1}}(x)\right| \geq\left|\operatorname{GRD}_{\$_{1}}(x)\right|$, a contradiction.

- If $\beta_{3}=2$, then $\beta_{1}=c_{2}-2>k-2$. Thus, we have $\left|\mathrm{OPT}_{\$_{1}}(x)\right|>\left|\mathrm{GRD}_{\$_{1}}(x)\right|$, a contradiction.

Therefore, $\$_{3}=\left\langle 1, c_{2}, c_{3}\right\rangle$ is canonical.

$(\Leftarrow)$ First, we show that $\$_{1}=\left\langle 1,2, c_{3}, c_{3}+1\right\rangle$ is non-canonical with $c_{3}>3$. It is easy to see $\mathrm{OPT}_{\$_{1}}\left(2 c_{3}\right)=(0,0,2,0)$. By Theorem 1, we have $\operatorname{GRD}_{\$_{1}}\left(2 c_{3}\right)=\left(\alpha_{1}, \alpha_{2}, 0,1\right)$. Since $c_{3}>3$, we have $\left|\operatorname{GRD}_{\$_{1}}\left(c_{3}-1\right)\right|>1$, i.e., $\left|\operatorname{GRD}_{\$_{1}}\left(2 c_{3}\right)\right|>2$. Thus, $\$_{1}$ is non-canonical.

Secondly, we show that $\$_{2}=\left\langle 1,2, c_{3}, c_{3}+1,2 c_{3}\right\rangle$ is canonical. Assume that $\$_{2}$ is non-canonical. By Theorem 3, there is the smallest counterexample $x$ of $\$_{2}$ such that $x \in\left[c_{3}+2,3 c_{3}\right)$ and $x \neq 2 c_{3}$. Next, we will deduce a contradiction by analyzing $x$ in detail.

(1) If $x \in\left[c_{3}+2,2 c_{3}\right)$, then it is also the smallest counterexample of $\$_{1}$. For simplicity, let $x=c_{3}+\kappa$ with $\kappa \in\left[2, c_{3}\right)$.

- If $\kappa=2 \ell+1$, then $1 \leq \ell \leq \frac{c_{3}-1}{2}-1$. By Theorem 1, we have

$$
\begin{aligned}
\operatorname{GRD}_{\$_{1}}(x) & =(0, \ell, 0,1) \\
\mathrm{OPT}_{\$_{1}}(x) & =\left(\beta_{1}, \beta_{2}, 1,0\right)
\end{aligned}
$$

Thus, $\beta_{1}+2 \beta_{2}=2 \ell+1$ and $\beta_{1} \in\{0,1\}$. It is easy to see $\left|\mathrm{GRD}_{\$_{1}}(x)\right|<\left|\mathrm{OPT}_{\$_{1}}(x)\right|$, a contradiction.

- If $\kappa=2 \ell$, then $2 \leq \ell \leq \frac{c_{3}}{2}-1$. Similarly, we have

$$
\begin{aligned}
\operatorname{GRD}_{\$_{1}}(x) & =(1, \ell-1,0,1) \\
\operatorname{OPT}_{\$_{1}}(x) & =(0, \ell, 1,0)
\end{aligned}
$$

Obviously, $\left|\mathrm{OPT}_{\$_{1}}(x)\right|=\left|\operatorname{GRD}_{\$_{1}}(x)\right|$, a contradiction.

(2) Otherwise, $x \in\left(2 c_{3}, 3 c_{3}\right)$. Let $x=2 c_{3}+\kappa$ with $\kappa \in\left[2, c_{3}\right)$.

- If $\kappa=2 \ell+1$, then $1 \leq \ell \leq \frac{c_{3}-1}{2}-1$. By Theorem 1, we have

$$
\begin{aligned}
\operatorname{GRD}_{\$_{2}}(x) & =(1, \ell, 0,0,1) \\
\operatorname{OPT}_{\$_{2}}(x) & =\left(0, \beta_{2}, \beta_{3}, \beta_{4}, 0\right)
\end{aligned}
$$

If $\beta_{3}+\beta_{4} \geq 3$, then $2 \beta_{2}+\beta_{3} c_{3}+\left(c_{3}+1\right) \beta_{4} \geq$ $3 c_{3}>x$, a contradiction.

And for $\beta_{3}+\beta_{4}=0, \beta_{3}+\beta_{4}=1$ and $\beta_{3}+\beta_{4}=2$, it is easy to get a contradiction similarly.

- If $\kappa=2 \ell$, then we can also deduce a contradiction similar to the above case.

Thus, $\$_{2}=\left\langle 1,2, c_{3}, c_{3}+1,2 c_{3}\right\rangle$ is canonical.

$(\Rightarrow)$ For the integer $c_{3}+c_{4}$, it is easy to see $\mathrm{GRD}_{\$_{2}}\left(c_{3}+c_{4}\right)=$ $\left(\alpha_{1}, \alpha_{2}, 0,0,1\right)$ and $\mathrm{OPT}_{\$_{2}}\left(c_{3}+c_{4}\right)=(0,0,1,1,0)$.
Since $\$_{2}$ is canonical, we have either $\alpha_{1}=1, \alpha_{2}=0$ or $\alpha_{1}=0, \alpha_{2}=1$, i.e.,

$$
c_{5}=c_{3}+c_{4}-1 \text { or } c_{5}=c_{3}+c_{4}-c_{2} .
$$

For the integer $2 c_{4}$, we have

$$
c_{5}=2 c_{4}-1 \text { or } c_{5}=2 c_{4}-c_{2} \text { or } c_{5}=2 c_{4}-c_{3}
$$

Correlating (A) with $(\mathrm{B})$, we have 3 feasible equations as follows:

$$
\text { (1) } \begin{gathered}
\left\{\begin{array} { l } 
{ c _ { 5 } = c _ { 3 } + c _ { 4 } - c _ { 2 } } \\
{ c _ { 5 } = 2 c _ { 4 } - c _ { 3 } }
\end{array} \text { (2) } \left\{\begin{array}{l}
c_{5}=c_{3}+c_{4}-1 \\
c_{5}=2 c_{4}-c_{3}
\end{array}\right.\right. \\
\text { (3) }\left\{\begin{array}{l}
c_{5}=c_{3}+c_{4}-1 \\
c_{5}=2 c_{4}-c_{2}
\end{array}\right.
\end{gathered}
$$

Next, we will deduce a contradiction from (1) and (2) respectively.

(1) Solving (1), we have $c_{4}=2 c_{3}-c_{2}$ and $c_{5}=3 c_{3}-2 c_{2}$. Thus,

$$
\$_{2}=\left\langle 1, c_{2}, c_{3}, 2 c_{3}-c_{2}, 3 c_{3}-2 c_{2}\right\rangle
$$

Since $\$_{1}$ is non-canonical, there is the smallest counterexample $x \in\left[c_{3}+2,3 c_{3}-c_{2}-1\right]$ of $\$_{1}$.

If $x \in\left[c_{3}+2,3 c_{3}-2 c_{2}-1\right]$, then $x$ is also a counterexample of $\$_{2}$, contradiction.

Otherwise, $x \in\left[3 c_{3}-2 c_{2}, 3 c_{3}-c_{2}-1\right]$. By Theorem 1 we have

$$
\begin{aligned}
& \operatorname{GRD}_{\$_{1}}(x)=\left(\alpha_{1}, \alpha_{2}, 0,1\right) \\
& \operatorname{OPT}_{\$_{1}}(x)=\left(\beta_{1}, \beta_{2}, \beta_{3}, 0\right) \quad \text { with } \beta_{3} \leq 2
\end{aligned}
$$

- If $c_{3} \geq 2 c_{2}$, then $\operatorname{GRD}_{\$_{1}}(x)=\left(\alpha_{1}, \alpha_{2}, 0,1\right)$ and $\mathrm{OPT}_{\$_{1}}(x)=\left(\beta_{1}, 0, \beta_{3}, 0\right)$ where $\beta_{3} \in\{0,1,2\}$ and $\alpha_{2}>0$. Since $x \in\left[3 c_{3}-2 c_{2}, 3 c_{3}-c_{2}-1\right]$, we have $\left|\operatorname{GRD}_{\$_{1}}(x)\right| \leq c_{3}-2 c_{2}+2$.

For $\beta_{3}=0, \beta_{3}=1$ and $\beta_{3}=2$, it is easy to deduce a contradiction respectively.

- If $c_{3}<2 c_{2}$, then $\operatorname{GRD}_{\$_{1}}(x)=\left(\alpha_{1}, 0,0,1\right)$ and $\mathrm{OPT}_{\$_{1}}(x)=\left(0, \beta_{2}, \beta_{3}, 0\right)$ where $\beta_{3} \in\{0,1,2\}$ and $\beta_{2}+\beta_{3}<1+\alpha_{1}$.

- If $\beta_{3}=2$, then $\beta_{2}=0$ and $\alpha_{1}=c_{2}$, a contradiction.

- If $\beta_{3}=1$, then $c_{3}=\left(\beta_{2}+1\right) c_{2}-\alpha_{1}$. Since $c_{3} \in\left(c_{2}, 2 c_{2}\right)$, we have $c_{3}=2 c_{2}-\alpha_{1}$. Thus,

$$
\$_{2}=\left\langle 1, c_{2}, 2 c_{2}-\alpha_{1}, 3 c_{2}-2 \alpha_{1}, 4 c_{2}-3 \alpha_{1}\right\rangle
$$

If $\alpha_{1}=1$, then $\$_{2}=\left\langle 1, c_{2}, 2 c_{2}-1,3 c_{2}-\right.$ $\left.2,4 c_{2}-3\right\rangle$. It is easy to see $\left\langle 1, c_{2}, 2 c_{2}-1,3 c_{2}-\right.$ $2\rangle$ is canonical, a contradiction.

If $\alpha_{1}>1$, then $4 c_{2}-2 \alpha_{1}$ is a counterexample of $\$_{2}$, a contradiction.

- If $\beta_{3}=0$, then $c_{3}=\frac{\beta_{2}-1}{2} c_{2}+\left(c_{2}-\frac{\alpha_{1}}{2}\right)=$ $q c_{2}+r$. Since $c_{3}<2 c_{2}$, we have $\beta_{2}=3$ and $x=3 c_{2}$ and $q=1, r=c_{2}-\frac{1}{2} \alpha_{1}$. Thus,

$$
\$_{2}=\left\langle 1, c_{2}, c_{2}+r, c_{2}+2 r, c_{2}+3 r\right\rangle
$$


Since $\beta_{2}+\beta_{3}<1+\alpha_{1}$, we have $\alpha_{1}>2$. By Theorem $4 \$_{3}=\left\langle 1, c_{2}, c_{2}+r\right\rangle$ is non-canonical. It is easy to see $2 c_{2}<c_{2}+2 r$ is the smallest counterexample of $\$_{2}$, a contradiction.

(2) Solving (2), we have $c_{4}=2 c_{3}-1$ and $c_{5}=3 c_{3}-2$. Thus,

$$
\$_{2}=\left\langle 1, c_{2}, c_{3}, 2 c_{3}-2,3 c_{3}-2\right\rangle
$$

It is easy to see $c_{3}+c_{4}-1<c_{5}$, i.e., the smallest counterexample of $\$_{1}$ is also a counterexample of $\$_{2}$, a contradiction.

(3) Solving (3), we have $c_{4}=c_{3}+c_{2}-1$ and $c_{5}=$ $2 c_{3}+c_{2}-2$. Thus,

$$
\$_{2}=\left\langle 1, c_{2}, c_{3}, c_{2}+c_{3}-1, c_{2}+2 c_{3}-2\right\rangle
$$

By Lemma 4, $\left\langle 1, c_{2}, c_{3}\right\rangle$ is canonical. Since $\$_{1}$ is noncanonical, $2 c_{3}$ is a counterexample of $\$_{1}$ by Theorem 2. And we claim $2 c_{3} \geq c_{2}+2 c_{3}-2$. Otherwise, $2 c_{3}$ is a counterexample of $\$_{2}$, a contradiction. Thus, $c_{2} \leq 2$, i.e.,

$$
\$_{2}=\left\langle 1,2, c_{3}, c_{3}+1,2 c_{3}\right\rangle
$$

This completes the proof.

Proof of Lemma 3. First, we introduce some notation as follows:

- $x=c_{m+1}+\delta$ is the smallest counterexample of $\$_{3}$.

- $c_{s}$ is the largest coin used in all the optimal representations of $x$ with $s \leq m$.

- $c_{l}$ and $c_{h}$ are the smallest coin and the largest coin used in the optimal representation of $x-c_{s}$ respectively.

Thus, we have $c_{l}>x-c_{s+1}$ by definition of $c_{s}$. Since $x$ is the smallest counterexample of $\$_{3}$, we have

$$
\begin{aligned}
\left|\operatorname{GRD}_{\$_{3}}\left(x-c_{s}\right)\right| & =\left|\operatorname{OPT}_{\$_{3}}\left(x-c_{s}\right)\right| \\
\left|\operatorname{GRD}_{\$_{3}}\left(x-c_{l}\right)\right| & =\left|\operatorname{OPT}_{\$_{3}}\left(x-c_{l}\right)\right| \\
\left|\operatorname{GRD}_{\$_{3}}\left(x-c_{h}\right)\right| & =\left|\operatorname{OPT}_{\$_{3}}\left(x-c_{h}\right)\right|
\end{aligned}
$$

Assume that $x$ is not the sum of two coins, i.e., $c_{s}+c_{l}<x$. Thus, we can find one coin $c_{h}$ besides a coin $c_{l}$ and a coin $c_{s}$ in the optimal representation of $x$.

(1) If $s \leq m-1$, then $c_{l}>x-c_{m}=\delta+d_{m+1}$. By Lemma 2 , it is easy to see $x-c_{h} \geq c_{s}+c_{l}>c_{s+1}$. Thus, $c_{s+1}$ should appear in the optimal representation of $x$, a contradiction.

(2) Otherwise, $s=m$.

- If $c_{h}+c_{m}>c_{m+1}$, there exists $c_{m+1}+c_{i}=c_{h}+c_{s}$ by the hypothesis. We can replace $c_{h}$ and $c_{m}$ with $c_{m+1}$ and $c_{i}$, a contradiction.

- If $c_{h}+c_{m}<c_{m+1}$, then $\mathrm{OPT}_{\$_{3}}(x)$ uses one coin $c_{l}$ besides a coin $c_{h}$ and a coin $c_{m}$. Since $s=m$, we have $c_{l}>x-c_{m+1}$, i.e., $\delta<c_{l} \leq c_{h}<d_{m+1}$. By the hypothesis, we have $c_{m+1}+c_{i}=2 c_{m}$. Consider $c_{m}+\delta<c_{m+1}$.

$$
\begin{aligned}
\left|\operatorname{GRD}_{\$_{3}}\left(c_{m}+\delta\right)\right| & =1+\left|\operatorname{GRD}_{\$_{3}}(\delta)\right| \\
& >1+\left|\operatorname{GRD}_{\$_{3}}\left(\delta+d_{m+1}\right)\right| \\
& =1+\left|\operatorname{GRD}_{\$_{3}}\left(c_{m}+\delta-c_{i}\right)\right|
\end{aligned}
$$

Therefore, $c_{m}+\delta$ is a counterexample of $\$_{3}$, a contradiction.

- If $c_{h}+c_{m}=c_{m+1}$, then $\mathrm{OPT}_{\$_{3}}(x)$ uses one coin $c_{l}$ besides a coin $c_{h}$ and a coin $c_{m}$. Thus, $\delta<c_{l} \leq$ $c_{h}=d_{m+1}$.

$$
\begin{aligned}
\left|\operatorname{GRD}_{\$_{3}}(x)\right| & =1+\left|\operatorname{GRD}_{\$_{3}}(\delta)\right| \\
& <2+\left|\operatorname{GRD}_{\$_{3}}(\delta)\right|=\left|\operatorname{OPT}_{\$_{3}}(x)\right|
\end{aligned}
$$

This is a contraction. 